EPJ Web of Conferences 92,02108 (2015)

DOI: $10.1051 /$ epjconf/ 20159202108

(C) Owned by the authors, published by EDP Sciences, 2015

\title{
An apparatus with a horizontal capillary tube intended for measurement of the surface tension of supercooled liquids
}

\author{
Václav Vinš ${ }^{1, a}$, Jan Hošek ${ }^{1}$, Jiří Hyk1 ${ }^{1}$ and Jan Hrubý ${ }^{1}$ \\ ${ }^{1}$ Institute of Thermomechanics AS CR, v. v. i., Dolejškova 1402, 18200 Prague 8, Czech Republic
}

\begin{abstract}
New experimental apparatus for measurement of the surface tension of liquids under the metastable supercooled state has been designed and assembled in the study. The measuring technique is similar to the method employed by P.T. Hacker [NACA TN 2510] in 1951. A short liquid thread of the liquid sample was sucked inside a horizontal capillary tube partly placed in a temperature-controlled glass chamber. One end of the capillary tube was connected to a setup with inert gas which allowed for precise tuning of the gas overpressure in order of hundreds of $\mathrm{Pa}$. The open end of the capillary tube was precisely grinded and polished before the measurement in order to assure planarity and perpendicularity of the outer surface. The liquid meniscus at the open end was illuminated by a laser beam and observed by a digital camera. Application of an increasing overpressure of the inert gas at the inner meniscus of the liquid thread caused variation of the outer meniscus such that it gradually changed from concave to flat and subsequently convex shape. The surface tension at the temperature of the inner meniscus could be evaluated from the overpressure corresponding to exactly planar outer meniscus. Detailed description of the new setup together with results of the preliminary tests is provided in the study.
\end{abstract}

\section{Introduction}

Surface tension of pure water and aqueous mixtures at low temperatures represents an important property both for scientific research [1] and industrial applications [2]. Water under the metastable supercooled state is currently investigated, especially, for better understanding and more accurate modelling of formation and growth of liquid droplets and ice crystals in upper atmosphere [3].

The surface tension of ordinary water under the stable conditions, i.e., at temperatures between the triple point and the critical point, can be described with correlation developed by Vargaftik et al. [4], approved subsequently as an international standard by IAPWS [5].

$$
\sigma=235.8 \tau^{1.256}(1-0.625 \tau)
$$

In equation (1), $\sigma$ is the surface tension in $\mathrm{mN} \mathrm{m}^{-1}, \tau$ denotes a dimensionless temperature $1-T / T_{\mathrm{c}}$ with critical temperature $T_{\mathrm{c}}=647.096 \mathrm{~K}$.

For the need of atmospheric modelling, the IAPWS correlation is usually extrapolated to temperatures below $0.01{ }^{\circ} \mathrm{C}$. However, correlation (1) was not derived using any data in the supercooled region. In the time of revision of the IAPWS correlation, only few data points for the surface tension of supercooled water were available; namely data by Humphreys and Mohler [6] from 1895 measured down to $-8{ }^{\circ} \mathrm{C}$, Hacker's data [7] from 1951, and rather scattered data by Floriano and Angel [8] from 1991.

Due to good internal consistency over relatively large temperature range, the data by Hacker [7] was considered as the most accurate until the recent past. However, Hacker's data shows a questionable change in trend at subzero temperatures. The surface tension increasing with decreasing temperature changes its slope approximately at a temperature of $-8{ }^{\circ} \mathrm{C}$. This anomaly, referred as the second inflection point in the temperature course of the surface tension of pure water, has been recently refuted by new measurements performed by our group [9]. The measuring technique was based on a modified capillary elevation method using a vertical capillary tube placed partly in a temperature-controlled chamber [10].

In this study, new experimental apparatus for measurement within a horizontal capillary tube is introduced. The measuring technique has similar principle as the approach developed by Ferguson and Kennedy [11] in 1932 which was later applied also by Hacker [7]. Measurements performed on the new apparatus might therefore help to clarify the existence of the second inflection point in Hacker's data. Moreover, new experiment shall represent a valuable proof of correctness of recently published data measured with the modified capillary elevation method.

\footnotetext{
${ }^{\text {a }}$ Corresponding author: vins@it.cas.cz
} 


\section{Experimental setup with a horizontal capillary tube}

Figure 1 shows a simplified scheme of the experimental apparatus developed in our laboratory. The core of the apparatus is represented by a horizontal capillary tube with inner diameter around $0.3 \mathrm{~mm}$ placed partly inside a specially designed glass chamber. One end of the capillary tube is left freely open to atmosphere, while the other end is connected to the setup distributing an inert gas. By applying small overpressure of gas, a sample of the investigated liquid sucked inside the capillary tube is pushed to the open end of the capillary tube. The liquid thread needs to be long such that the liquid meniscus inside the capillary tube is located within the glass chamber; see figure 1. The outer meniscus at the open end is illuminated by a laser beam and observed by an objective with a digital camera.

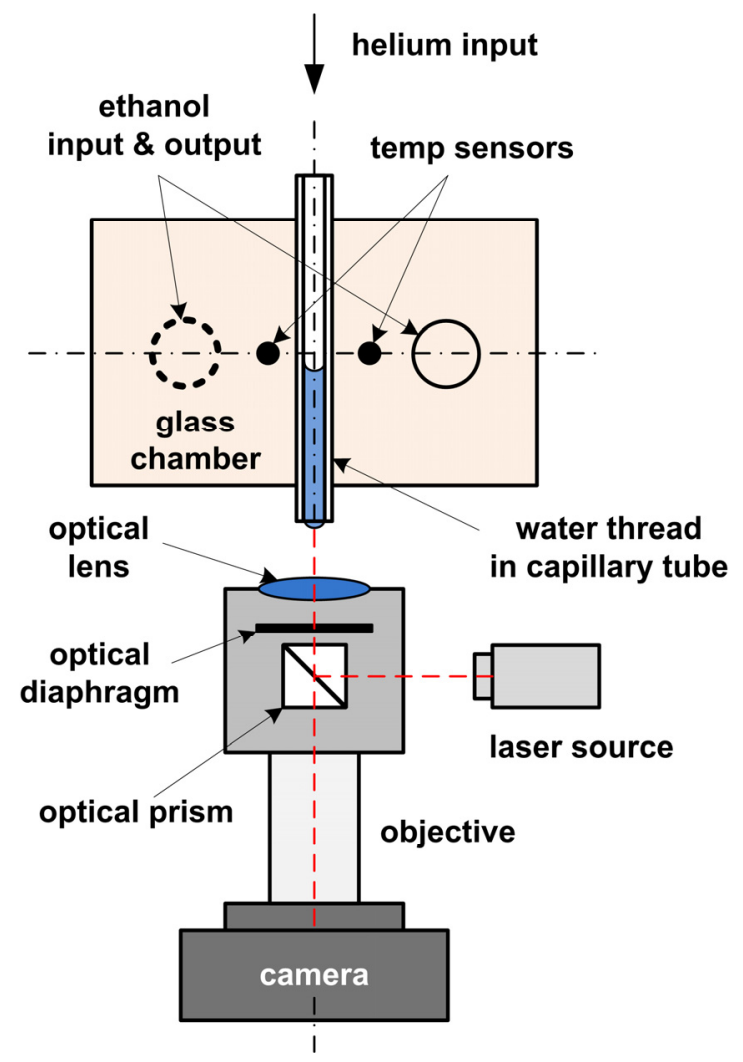

Figure 1. Scheme of the apparatus with the horizontal capillary tube; view from top

The glass chamber is connected to the temperaturecontrol unit consisting of two thermostatic baths operating with a mixture of ethanol and 1-propanol, silicon tubing, and a set of switching ball valves. One of the baths operates at temperature close to ambient, while the other one is set to the desired measuring temperature. The option of using two thermostatic baths allows fast variation of the temperature within the glass chamber which subsequently helps to reach lower degrees of supercooling of the liquid sample. The temperature inside the glass chamber is measured by two self-calibrated resistive thermometers Pt100 located in a position close to the capillary tube.
During the measurement, the overpressure of the inert gas is continuously varied in order to change the shape of the liquid meniscus at the open end of the capillary tube. Increasing overpressure results gradually in concave, flat, and convex shape of the outer meniscus. The surface tension of the liquid sample at a temperature within the glass chamber can be evaluated from the gas overpressure necessary for flattening the outer meniscus. In this case, the overpressure reaches equilibrium with surface forces over the inner meniscus only, as the flattened outer meniscus does not contribute to the force balance. Equation (2) is a simple formula derived from the YoungLaplace equation

$$
\sigma=\frac{\Delta p \cdot d}{4 \cos \theta}
$$

where, $\sigma$ stands for the surface tension, $\Delta p$ marks the overpressure, $d$ is the inner diameter of the capillary tube, and $\theta$ denotes the contact angle between the liquid and the capillary tube wall.

Photo of main parts of the setup is shown in figure 2 .

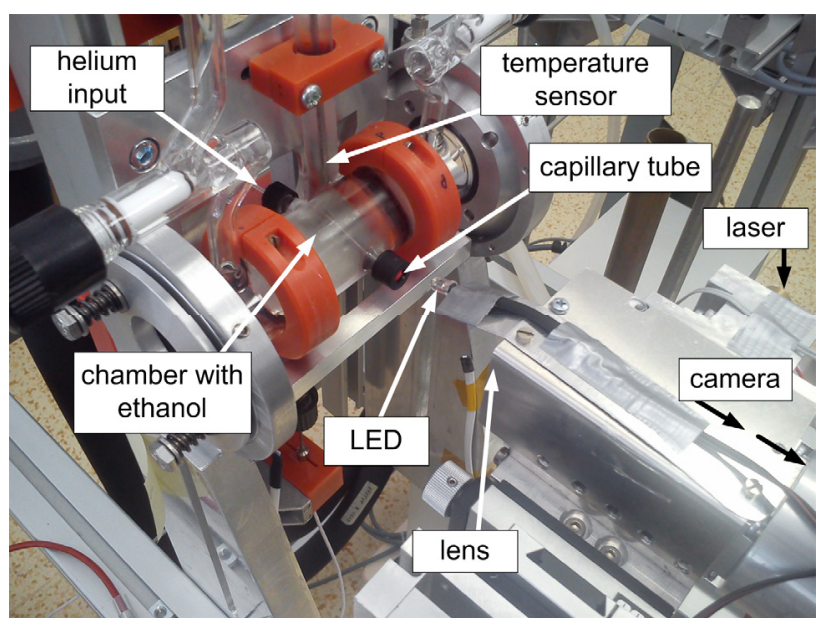

Figure 2. Photo of the central part of the experimental setup before installation of the thermal insulation on the glass chamber

\subsection{Capillary tube}

A quartz glass, i.e., fused silica, capillary tube with inner diameter around $0.3 \mathrm{~mm}$ and length of $7.5 \mathrm{~cm}$ has been used for our measurements. In order to assure physically proper behaviour of the outer liquid meniscus, the capillary tube open end required special treatment before the measurement. The open end needed to be precisely grinded and polished such that the outer surface was planar and perpendicular to the main capillary tube axis [12]. Moreover, to remove impurities on the inner surface and to avoid polluting aerosol particles from ambient air, the capillary tube was repeatedly flushed with chromosulfuric acid and pure water before each test.

The inner diameter of the capillary tube was derived in an indirect way from the surface tension measurements at positive temperatures and the IAPWS correlation (1) in the following way

$$
d=\frac{1}{N} \sum_{i=1}^{N} \frac{\Delta p\left(T_{i}\right) \cdot \sigma_{\mathrm{IAPWS}}\left(T_{i}\right)}{4 \cos \theta},
$$


where $N$ is the number of measurements at temperatures $T_{i}>0.01^{\circ} \mathrm{C}$ and $\theta$ is a constant contact angle for water and fused silica equal to $3^{\circ}$ [13]. The average inner diameter of the capillary tube employed in the current study was evaluated as $d=0.3317 \pm 0.0020 \mathrm{~mm}$.

\subsection{Helium distribution setup}

Helium 4 was chosen as the operating gas in this study due to its low solubility in water compared to other gases. Moreover, helium shows low adsorption on the water liquid surface and consequently has negligible effect on the surface tension. Small overpressure of helium in order of hundreds of $\mathrm{Pa}$ was tuned by using distribution setup depicted in figure 3 .

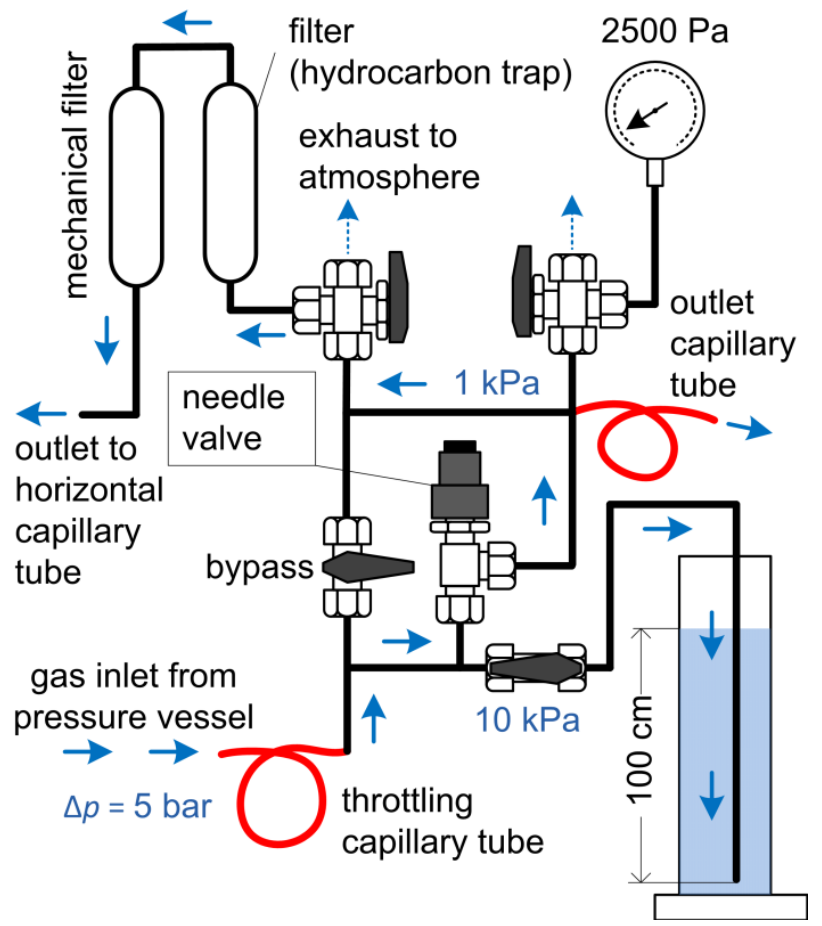

Figure 3. Helium distribution setup modified for measurement with the horizontal capillary tube

High pressure gas supported from the pressure vessel was throttled down to absolute pressure of $111 \mathrm{kPa}$ using a stainless steel capillary tube with inner diameter of $0.25 \mathrm{~mm}$ and length of $1.50 \mathrm{~m}$. The overpressure of $10 \mathrm{kPa}$ behind the capillary tube was defined by a gas flow through a water column of 1 meter height. The setup was designed such that the total maximum flow rate of helium did not exceed $200 \mathrm{~cm}^{3} \mathrm{~min}^{-1}$. The final low overpressure could be varied by changing the flow rate through the high-precision needle valve. An exhaust capillary tube with inner diameter of $0.25 \mathrm{~mm}$ and length of $3 \mathrm{~cm}$ was placed after the needle valve. The maximum overpressure of helium within this section could reach $1500 \mathrm{~Pa}$ at a maximum flow rate of $15 \mathrm{~cm}^{3} \mathrm{~min}^{-1}$. The setup was equipped with a set of filters in order to remove any mechanical and organic impurities from the gas which could consequently affect the liquid meniscus. The helium overpressure was measured by a differential pressure transducer Furness Control FC0332 with the range of $0 \div 2500 \mathrm{~Pa}$. The overpressure was measured with uncertainty better than $\pm 3.0 \mathrm{~Pa}$.

The setup shown in figure 3 was prepared by the modification of the previous system employed during measurements with the vertical capillary tubes [10].

\subsection{Temperature-controlled chamber}

Glass chamber with inner diameter of $30 \mathrm{~mm}$ is equipped with thread necks closed with open-top caps fitted with Teflon-silicon rubber septa penetrated by the fused silica capillary tube. Two in-house calibrated temperature sensors connected to a digital thermometer ASL F500 measure the temperature of alcohol mixture in the region close to the capillary tube. The uncertainty of measured temperature was better than $\pm 0.06^{\circ} \mathrm{C}$.

\subsection{Optics observing the meniscus}

The outer meniscus of the liquid thread inside the capillary tube was illuminated by a red laser. The laser beam from the laser source was directed on the meniscus with an optical prism and a lens. The image of the meniscus was observed by an objective and a digital camera Thorlabs DCC. All optical components were installed on the $x-y-z$ levelling table. Simplified scheme of the optical setup is shown in figure 1 .

A light-emitting diode (LED) with an additional voltage output was attached to the optical setup. Switching LED on and off allowed synchronization of the video record with other logged quantities, i.e. temperature and pressure.

\section{Preliminary measurements}

New experimental apparatus was successfully tested on measurements with pure water. The surface tension of ordinary water was measured at temperatures down to $-7{ }^{\circ} \mathrm{C}$ during preliminary tests. Ultrapure water with constant resistivity of $18.2 \mathrm{M} \Omega \mathrm{cm}$, total organic carbon of $1 \div 5 \mathrm{ppb}$, and free of particles larger than $0.2 \mu \mathrm{m}$ was used in the measurement.

\subsection{Capillary tube filling}

A proper and well reproducible filling of the capillary tube with a liquid sample represents an important step during preparation of the measurement. An unfavourable issue is the fact that, after the cleaning with acid and pure water, any contact of the inner surface of the capillary tube with polluting ambient air shall be avoided.

Thanks to relatively high surface tension under the ambient conditions, the sample of liquid such as water flows rather fast into the small diameter capillary tubes. Consequently, it is almost impossible to achieve a reproducible liquid thread of a defined length of several centimetres under ambient pressure inside the capillary tube.

Unfortunately, the option of pushing the liquid sample out of the capillary tube by increasing overpressure on 
the other side of the tube does not work either. Due to the high demand on cleanliness of the capillary tube inner surfaces, the liquid thread runs abruptly out of the capillary tube in the moment when the applied overpressure exceeds the surface forces at the liquid meniscus inside the capillary tube.

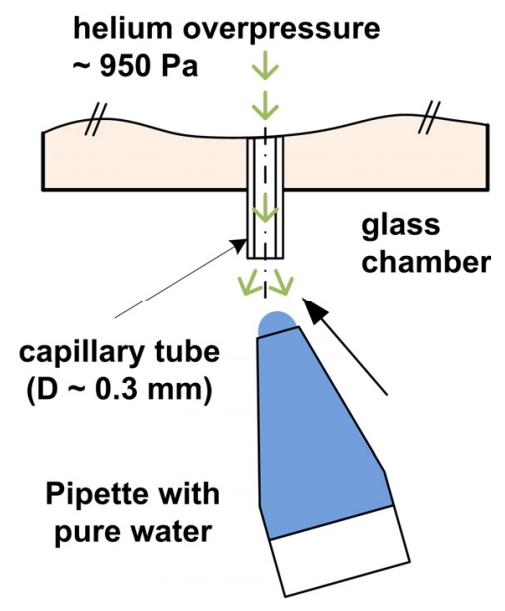

Figure 4. Filling of the horizontal capillary tube

After several unsatisfactory tests described above, following procedure was found to lead to the suitable and reproducible capillary tube filling; see figure 4. A well defined overpressure of helium shall be applied to the closed capillary tube immediately after its cleaning. The overpressure needs to be approximately $10 \%$ higher than the equilibrium overpressure corresponding to the surface forces at the meniscus inside the capillary tube at the ambient temperature, e.g., for water inside the capillary tube with inner diameter of $0.33 \mathrm{~mm}$ and ambient temperature $+25^{\circ} \mathrm{C}$, the equilibrium overpressure and the overpressure for filling shall approximately be $870 \mathrm{~Pa}$ and $950 \mathrm{~Pa}$, respectively. With freely open end of the capillary tube, the actual pressure at the exhaust is much smaller than the equilibrium overpressure. As soon as the pipette with water sample is attached to the open end of the capillary tube, the surface forces at the inner meniscus balance the overpressure inside the tube which continuously increases to the pre-set value $(950 \mathrm{~Pa})$. Unlike other attempts, this procedure results in liquid thread of a well reproducible length, i.e., $2 \mathrm{~cm}$ in our case.

\subsection{Pressure measurement}

The differential pressure transducer was connected to the data acquisition unit (DAQ) Agilent 34970A with scanning period of $125 \mathrm{~ms}$. In order to synchronize the pressure data with the recorded video the LED was turned off and subsequently on few seconds after and before the measurement start and measurement end, respectively. The pressure was gradually twice increased and decreased during each run. Figure 5 shows typical example of the recorded pressure over time at temperature $+25.3{ }^{\circ} \mathrm{C}$. Two full periods of the moving meniscus helped to detect potential errors in the measurement. The average overpressure corresponding to flat meniscus was $865.5 \mathrm{~Pa}$ in this case.

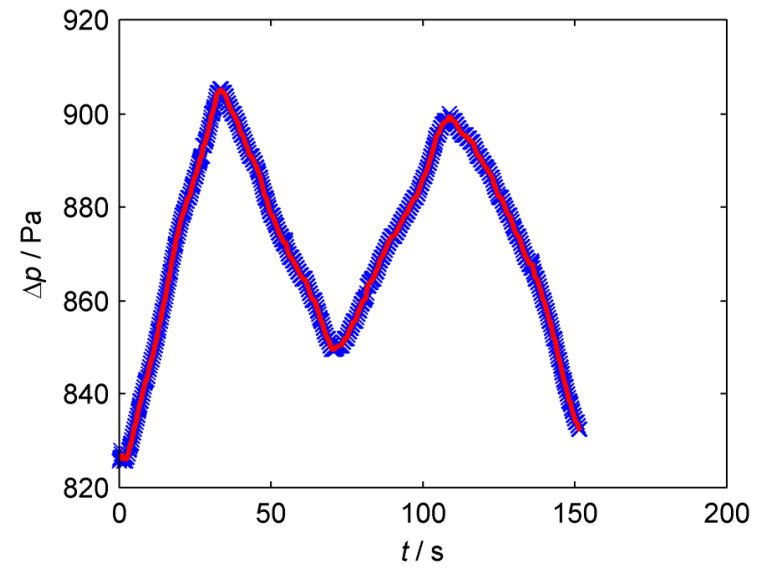

Figure 5. Pressure trend recorded during two periods of the oscillating water meniscus at temperature of $+25.3{ }^{\circ} \mathrm{C} ; \mathrm{x}$, recorded pressure; -, moving average of the recorded pressure

\subsection{Analysis of the video}

A constant frame rate of 8 frames per second was set on the digital camera observing the liquid meniscus. Unlike in case of the pressure measurement, the video frequency was slightly varying during the measurement. Consequently, the video time had to be interpolated to agree with the DAQ time. Voltage signal from LED was successfully used for this purpose.

Unlike convex or concave meniscus, the flat meniscus has a uniform distribution of light intensity. As soon as the meniscus becomes non-planar the light intensity significantly increases in the centre of the meniscus whose behaviour can be compared to a curved mirror. The planarity of the meniscus could therefore be analyzed in a sense of comparing the total relative deviation of the light intensity from its median. For planar meniscus, the deviation from the intensity median becomes minimum.

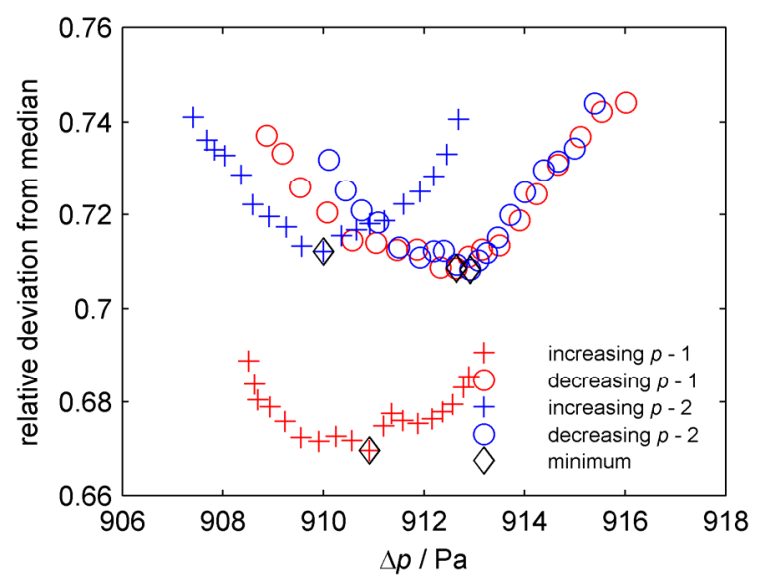

Figure 6. Example of relative deviation from median of intensity depending on helium overpressure at $+3.1{ }^{\circ} \mathrm{C}$. Minimum corresponds to the flat meniscus with uniform distribution of intensity.

Figure 6 shows an example of relative deviation of the light intensity from its median depending on recorded pressure. Depicted minima correspond to flat meniscus during two periods of varying helium pressure. In case of 
successful measurement, the pressure minima lie close to each other with a standard A-type uncertainty below $\pm 1.0 \mathrm{~Pa}$.

More details about the optical setup and the analysis of the recorded video are provided in Hošek et al. [14].

\section{Results and discussion}

The surface tension of water was measured with varying temperature steps such that the measurement at a temperature close to ambient was followed by measurement at low temperature and vice versa. Such approach assured that each measurement at a given temperature was independent of the previous measurements. Two independent data points were collected at each temperature in order to detect and avoid potential errors in the recorded video and pressure. Figure 7 shows the overpressure of helium depending on temperature measured inside the glass chamber. As can be seen, the overpressure compensating the surface tension of water at the inner meniscus increased by $100 \mathrm{~Pa}$ by temperature decrease from $+40^{\circ} \mathrm{C}$ to $-7^{\circ} \mathrm{C}$.

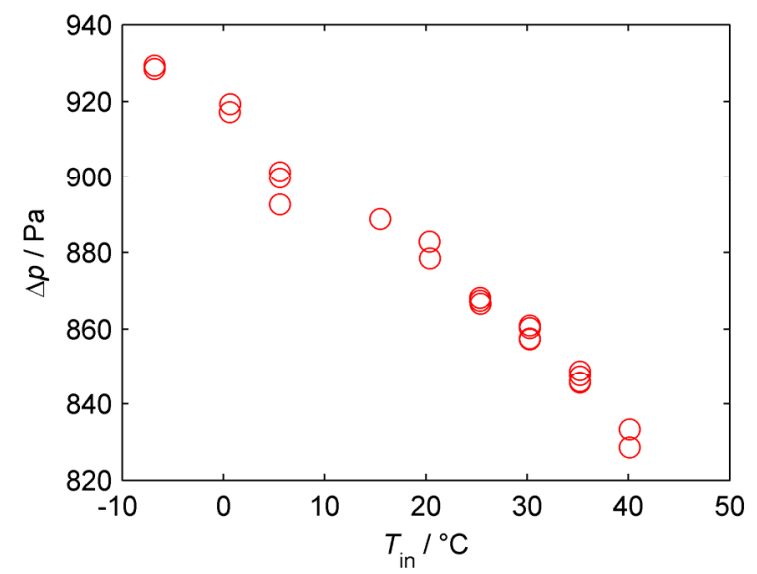

Figure 7. Overpressure of helium corresponding to the flat meniscus measured at various temperatures.

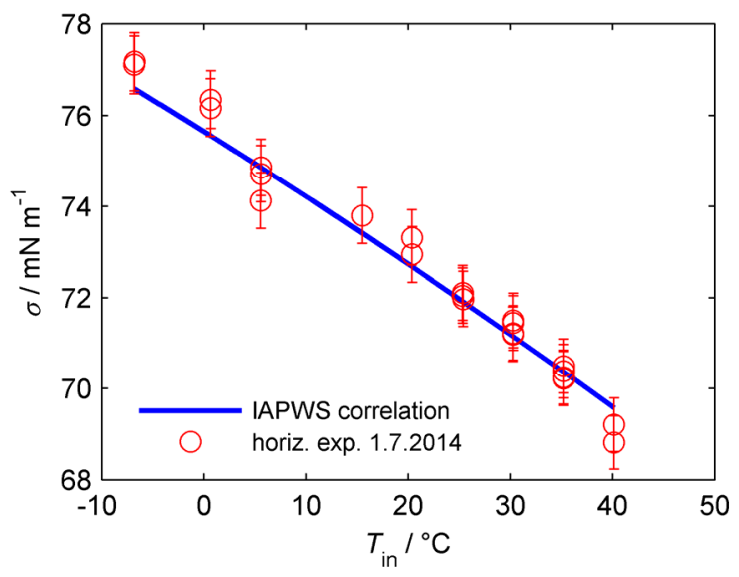

Figure 8. Preliminary results for the surface tension of ordinary water measured using horizontal apparatus compared to IAPWS correlation extrapolated to subzero temperatures.
The surface tension of water under both stable and metastable supercooled state evaluated from equation (2) is shown in figure 8. Preliminary data agree relatively well with the IAPWS correlation (1) extrapolated to subzero temperatures and consequently also to our previous results obtained with the capillary elevation method $[9,10]$. The estimated standard combined uncertainty of the measured surface tension is approximately 1.5 times higher than for previous measurements (around $\pm 0.40 \mathrm{mN} \mathrm{m}^{-1}$ at $-4{ }^{\circ} \mathrm{C}$ in [9]) as it increases from $\pm 0.59 \mathrm{mN} \mathrm{m}$ at $+40{ }^{\circ} \mathrm{C}$ to $\pm 0.63 \mathrm{mN} \mathrm{m}^{-1}$ at $-7^{\circ} \mathrm{C}$. Main reason is rather high uncertainty of the evaluated helium overpressure which shall be improved in future. As can be seen in figure 8 , the IAPWS correlation lies within the uncertainty range for most of the measured data points.

\section{Conclusions}

A new experimental apparatus intended for measurement of the surface tension of pure liquids under the metastable supercooled conditions has been introduced in the study. The apparatus is based on measurement within a short horizontal capillary tube placed partly inside a temperature-controlled chamber. The outer meniscus of the liquid thread can be flattened by applying a well defined overpressure of helium to the second meniscus inside the capillary tube. Planarity of the outer meniscus is detected by an optical setup consisting of a laser source and a digital camera with an objective [14].

All main components of the new apparatus were successfully tested. Preliminary data for the surface tension of water measured down to $-7{ }^{\circ} \mathrm{C}$ agrees with the IAPWS correlation extrapolated below $0.01{ }^{\circ} \mathrm{C}$ [5] and with our previous measurements performed with the vertical capillary tubes [9].

In future, the setup will be further improved in order to achieve higher degrees of supercooling and to improve the uncertainty of the measured data. Measurements made with new experimental technique shall on one hand support correctness of our previous research and on the other hand help to clarify the existence of the second inflection point in the data for the surface tension of supercooled water obtained by Hacker [7] who employed similar experimental method.

\section{Acknowledgement}

The study was conducted with the help of the institutional support RVO:61388998 from IT AS CR and partly also from the grant M100761201 from the Academy of Sciences of the Czech Republic. Many thanks go also to prof. K. Studenovský from the Czech Technical University in Prague for his help with the capillary tube grinding and polishing.

\section{References}

1. M.E. Earle, T. Kuhn, A.F. Khalizov, J.J. Sloan, Atmos. Chem. Phys. 10, 7945 (2010) 
2. S. Fernández-González, J.L. Sánchez, E. Gascón, L. López, E. García-Ortega, A. Merino, A. Sci. World J. 279063 (2014)

3. M.A.L.J. Fransen, E. Sachteleben, J. Hrubý, D.M.J. Smeulders, Exp. Fluids 55, 1780 (2014)

4. N.B. Vargaftik, B.N. Volkov, L.D. Voljak. J. Phys. Chem. Ref. Data 12, 817 (1983)

5. IAPWS release on surface tension of ordinary water substance. 1994; http://www.iapws.org

6. W.J. Humphreys, J.F. Mohler, Phys. Rev. 2, 387 (1895)

7. P.T. Hacker, NACA TN 2510 (1951)

8. M.A. Floriano, C.A. Angell, J. Phys. Chem. 94, 4199 (1990)

9. J. Hrubý, V. Vinš, R. Mareš, J. Hykl, J. Kalová, J. Phys. Chem. Lett. 5, 425 (2014)

10. V. Vinš, J. Hrubý, J. Hykl, J. Blaha, B. Šmíd, EPJ Web Conf. 45, 01094 (2013)

11. A. Ferguson, S.J. Kennedy, Proc. Phys. Soc. 44, 511 (1932)

12. J. Hošek, K. Studenovský, EPJ Web of Conf. 48, 00005 (2013)

13. V.G. Stepanov, L.D. Volyak, Y.V. Tarlakov, J. Eng. Phys. Thermophys. 32, 646 (1977) Translated from Inzhenerno-Fizicheskii Zhurnal 32, 1000 (1977)

14. J. Hošek, V. Vinš, J. Hykl, to be presented at Optics and Measurement International conference, 7-10 October 2014, Liberec, Czech Republic 